\title{
A Generalizing Fuzzy Model for Shallow Cavity Flows Under Different Mach Regimes ${ }^{1}$
}

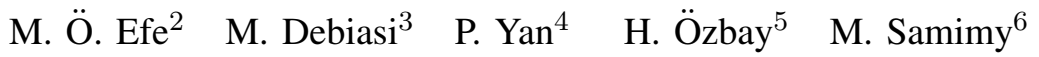

\begin{abstract}
Modeling of a flow passing over a shallow cavity is an interesting problem as the internal dynamics are inextricably intertwined due to the Navier-Stokes equations. The origin of the cavity flow identification problem is the desire for reducing the skin friction in aerial vehicles through an active control effort. As there is not a well developed closed-loop scheme, towards the goal of feedback control, the first step becomes to develop a suitable dynamic model imitating the behavior of the plant under certain operating conditions. For this purpose, we present an approach exploiting the fuzzy inference mechanisms. Fuzzy logic is a practical tool for expressing human expertise in the form of if-then statements. The idea in the fuzzy identification is to perform local observations from the flow field and to find a fuzzy interpolation scheme over the sensory information. The results we have observed indicate that a classical non-adaptive fuzzy model is able to perform one step ahead prediction of the critically essential behavior observed at the cavity floor.
\end{abstract}

\section{INTRODUCTION}

Fuzzy Logic (FL) is a practical framework for solving complicated problems by utilizing expert knowledge. The practicality of the paradigm stems from the fact that the human expertise is expressed in the form of IF antecedent THEN consequent statements, i.e. the task to be achieved is modeled through the use of linguistic descriptions. Since the early works of Zadeh on fuzzy systems, the methodology has become a well-developed framework and enjoyed by a wide spectrum of applications.

The typical architectures of fuzzy inference systems are those introduced by Wang [1-2], Takagi and Sugeno [3] and Jang et al. [4]. In [1], a fuzzy system having Gaussian membership functions, product inference rule and weighted average defuzzifier is constructed and has become the standard method in most applications. Takagi and Sugeno change

\footnotetext{
${ }^{1}$ This work was supported in part by AFRL/VA and AFOSR under contract no F33615-01-2-3154 and in part by the European Commission under contract no. MIRG-CT-2004-006666.

${ }^{2}$ Corresponding Author, TOBB University of Economics and Technology, Department of Electrical and Electronics Engineering, Sögütözü, Ankara, Turkey, E-mail: onderefe@ieee.org, Phone: +90-312-292-4064, Fax: +90312-292 4091

${ }^{3}$ Department of Mechanical Engineering, The Ohio State University, Columbus, OH 43210, U.S.A., E-mail: debiasi.1@osu.edu

${ }^{4}$ was with Department of Electrical and Computer Engineering, The Ohio State University, Columbus, $\mathrm{OH}$ 43210, USA; present address: Seagate Tech Center, Bloomington MN 55435 USA, E-mail: yan.39@osu.edu, Peng.Yan@seagate.com

${ }^{5}$ Department of Electrical and Electronics Engineering, Bilkent University, Bilkent, TR-06800, Ankara, Turkey, on leave from Department of Electrical and Computer Eng., The Ohio State University, E-mail: hitay@bilkent.edu.tr, ozbay@ece.osu.edu

${ }^{6}$ Department of Mechanical Engineering, The Ohio State University, Columbus, OH 43210, U.S.A., E-mail: samimy.1@ osu.edu
}

the defuzzification procedure where dynamic systems are used in the defuzzification stage. The potential advantage of the Takagi-Sugeno fuzzy models is that under certain constraints, the stability of the system can be studied [5-6]. Jang et al., [4], propose an adaptive neuro-fuzzy inference system, in which a polynomial is used as the defuzzifier. This structure is commonly referred to as ANFIS in the related literature. The choice concerning the order of the polynomial and the variables to be used in the defuzzifier are left to the designer.

In [7], Cohen et al. use FL for the control of a circular cylinder vortex shedding model. The fuzzy system in [7] has been used to scale a control signal produced by a PID controller, and it has been shown that such a strategy yields significant improvement in the performance compared to the sole PID solution. In [8], FL with triangular membership functions is used for controlling the vortex flows on a generic X29-A model. The fuzzy controller is compared with neural controllers and predictive schemes. Dragojlovic et al. utilize the fuzzy logic in improving the performance of a Computational Fluid Dynamics (CFD) solver. The fuzzy control scheme guides the increment in the relaxation factor by using triangular membership functions, [9-10]. Depending on the past solution entries, the CFD solver automatically adjusts itself to exploit the best relaxation factor. Efe et al. demonstrate that FL can be used in frequency domain quantification and a model interpolation example is studied for Burgers equation, [11].

Although the fuzzy systems can be used in a wide variety of applications of flow problems, as the number of variables involved increases, it becomes a tedious task to build an efficient rule base leading to a useful fuzzy model. In this study, we consider the fuzzy inference systems as a tool for identifying the phenomenon taking place inside the experimental facility. The motivating factors for utilizing the fuzzy logic for the problem at hand can be summarized as follows: Firstly, the parameters of the continuous time form of the flow field are not measurable as the system dynamics is given by Partial Differential Equations (PDEs) This forces us to develop sampled-data based techniques. Secondly, the available data is in discrete time and localized, i.e. finitely many sensory information is available. Thirdly, there is not a dynamic model for the actuation device(s) and the sensors. All the necessary information regarding the system and its components are hidden in the numerical data. Lastly, the observed data contains uncertainties caused by measurement imperfections. These facts naturally point out to exploit techniques that can yield useful models based on 
the numerical observations, that can easily incorporate the human expertise into the design and that are suitable for post-design interpretation.

As outlined above, some work has been done in the past decade to explore the use of fuzzy logic in flow systems with some degrees of success. Several of these works showed promising results but were based on numerical simulations and lacked any experimental validation of the concept. To our best knowledge, no attempt has been made so far in using FL to model a dynamic, higher frequency flow like the one over a cavity. Therefore still many questions remain open about the merit and effectiveness of fuzzy inference systems in flow modeling and control. Having this motivation in mind, in this paper, we work on the experimental setup shown in Figs. 1-2 and introduced in the next section. The goal is to characterize the flow passing over a cavity based on local pressure measurements. The methodology is presented in the next section, and the obtained results are discussed in the third section. The conclusions constitute the last part of the paper.

\section{THE EXPERIMENTAL FACILITY}

In this study, the experimental facility illustrated in Fig. 1 and described in detail in [12-13] is used. The facility consists of an optically accessible, blow-down type wind tunnel capable of continuous operation in the subsonic range. A shallow cavity with a depth $D=12.74 \mathrm{~mm}$ and length $L=50.8 \mathrm{~mm}$ and having length to depth aspect ratio $\mathrm{L} / \mathrm{D}=$ 4 is recessed in the floor of the test section. The cavity shearlayer is forced by a 2-D synthetic-jet type actuator issuing from a high-aspect-ratio converging nozzle embedded in the cavity leading edge. Pressure fluctuations are measured by Kulite type dynamic pressure transducers placed in different locations in the test section and used to derive different acoustic quantities as described in Debiasi et al., [13]. The control flow is provided by a synthetic jet exhausting from a slot spanning the width of the cavity upstream wall (See Fig. 1). The flow control setup is equipped with a computer, which is capable of real-time acquisition of data from the pressure transducers or hot wires, and is able to actuate a synthetic actuator to affect the flow field in the test section. A data acquisition software with relevant Digital Signal Processor (DSP) board is installed on the host computer running the controller that closes the loop. As seen in Fig. 2 , the side windows of the experimental setup enable the operator to visualize the flow field inside the test section utilizing advanced laser-based techniques developed at Gas Dynamics and Turbulence Laboratory (GDTL) of OSU. Flow imaging techniques can be used to capture the instantaneous and phase-averaged features of the flow as described in [13] and [14]. The simultaneous time traces collected from the pressure transducers have been used to obtain a fuzzy model. It is critically important to emphasize that the collected data must be spectrally rich enough to capture the cases that are likely to be encountered in real-time operation. This makes it sure that the fuzzy model responds appropriately to the input variables.

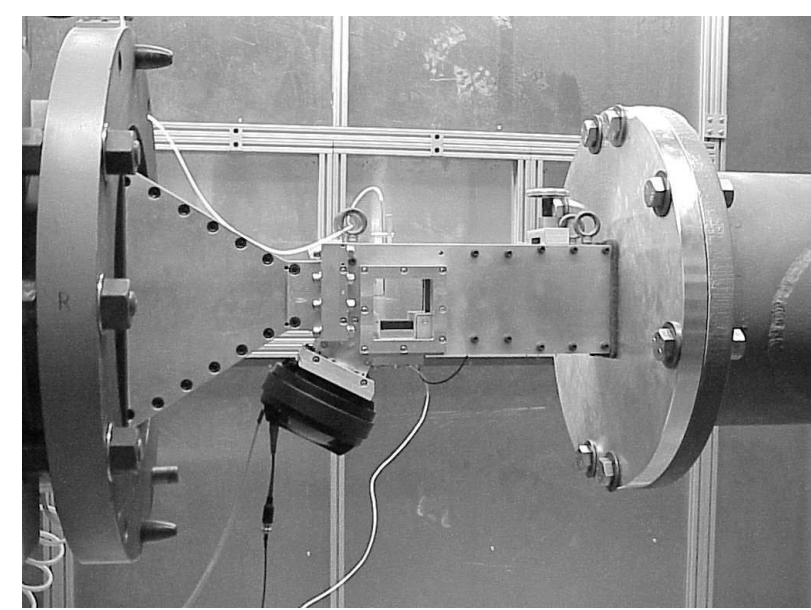

Fig. 1. The appearance of the overall system

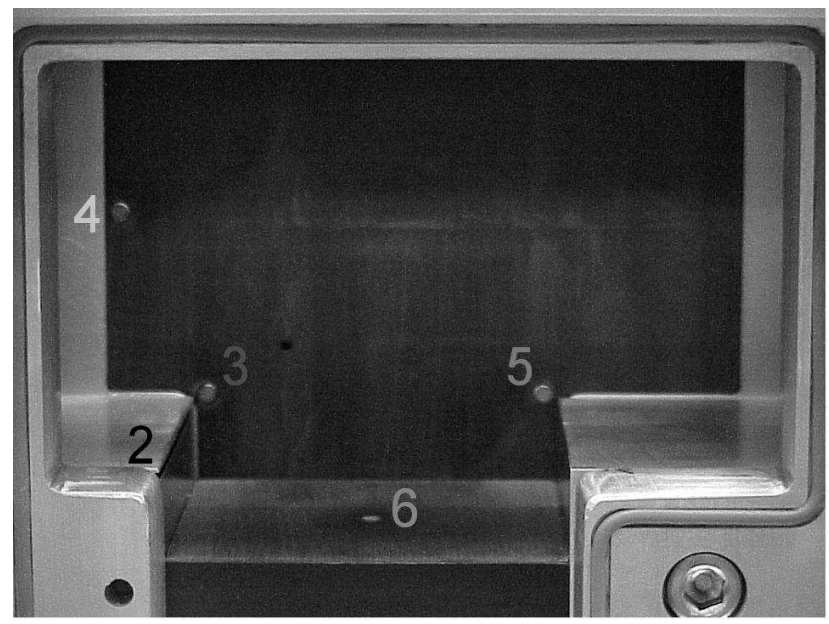

Fig. 2. The locations of the transducers placed in the test section

In [13], it is observed that the cavity flow exhibits strong, single-mode resonance in the Mach number ranges 0.25-0.31 and 0.39-0.5, and multi-mode resonance in the Mach number range $0.32-0.38$. In the same study, they also observed that the frequency of sinusoidal forcing with the synthetic jet-like actuator has a major impact on the cavity flow resonance whereas the effect of the amplitude is relatively minor and it affects the control authority only at higher Mach numbers. This prompted the development of a logic-based type of control that searches the forcing frequencies in a closedloop fashion that reduce the cavity flow resonant peaks and then maintains the system in such conditions through an open-loop control. The technique performed well in the experimental trials and allowed identification of optimal frequencies for the reduction of resonant peaks in the Mach number range 0.25-0.5.

Some linear feedback controllers have also been developed 
for subsonic cavity flows, [14-15]. Experimental results summarized in [15] have offered two important conclusions: (i) all the linear controllers derived from a linear plant model for a single dominant Rossiter mode were able to suppress the cavity oscillations at this mode, but they shift the oscillations to another Rossiter frequency, which was not present explicitly in the unforced case; and (ii) adding a zero to the simplest of these linear controllers, the proportional controller, avoids this problem, provided that the location of the zero matches the newly excited Rossiter mode mentioned above. The real time implementations on DSP board (See [15]) showed in particular that the parallel proportional controller with a time delay block outperforms the other linear controllers (classical PID controller, Smith predictor and H-Infinity controller) in terms of elimination of the main frequency of oscillation and robustness with respect to different Mach numbers. The resonant peak reduction is comparable to that obtained with logic based control, but the method is more robust with respect to slight changes in flow parameters. Recent works of Efe et al., [16-17], on the mentioned cavity flow has demonstrated that the flow behavior can be identified by neural networks. The feedback control research using neural networks is underway.

These simple yet effective control techniques represent a reference against which alternative control strategies could be compared, e.g. neuro/fuzzy controllers or controllers that exhibit some degree of autonomy and intelligence. Nevertheless, the availability of a dynamic model often precedes the controller design and this fact motivates us to approach the problem systematically from the computational intelligence point of view. In the next section, we explain the adopted fuzzy model development strategy. Since the experimental facility enables us to acquire pointwise observations from the physically critical locations of the cavity, one could use this information for identification and control of the cavity flow.

\section{FuZZy InfEREnCE Systems And Modeling}

\section{A. Generating the Experimental Data}

As can be seen from Fig. 2, simultaneous time traces can be obtained from the flow field. The sensors are located in such a way that the critical information about the flow physics is dense, i.e. the entry of control excitation, the upstream and downstream wall neighborhood, test section entry (baseline flow) and the cavity floor. In the tests, we have chosen the data read from the first, third and the sixth sensors shown in Fig. 2. Although it is not seen, the first sensor label is reserved for the sent excitation signal recorded within the host computer. Define the following variables;

$S_{1}$ measures $u_{1, k}$, the actuation signal in volts

$S_{2}$ measures $u_{2, k}$, the pressure fluctuations just before the actuator exit,

$S_{3}$ measures $u_{3, k}$, the pressure fluctuation just after the actuator exit (i.e. at the receptivity region at the cavity leading edge),

$S_{4}$ measures $u_{4, k}$, the pressure fluctuations (if any) before the cavity,

$S_{5}$ measures $u_{5, k}$, the pressure fluctuations at the cavity trailing edge,

$S_{6}$ measures $d_{k}$, the pressure fluctuations at the center of the cavity floor.

According to these definitions, we performed a set of experiments to collect the data. The first set of experiments address the noise driven cases. For Mach numbers equal to $0.25,0.28,0.30,0.32$ and 0.35 , we have excited the flow field with computer generated noise signal. The excitation signal is lowpass filtered with cutoff frequency $200 \mathrm{~Hz}$ and highpass filtered with cutoff frequency $10 \mathrm{kHz}$. This is implemented to meet the operating conditions of the synthetic actuator.

The second set of experiments includes open-loop free forced observations at the same Mach numbers stated above. The flow field is excited with sinusoidal signals of amplitude $2.35 V_{\mathrm{rms}}$ and frequency $3250 \mathrm{~Hz}$ and then another sinusoidal signal with $4.06 V_{\mathrm{rms}}$ magnitude and frequency equal to 3920 Hz. These numbers have been set according to the open loop expertise gained through the design of the experimental facility.

In the third set, we closed the loop with the previously tested controllers reported in [13-15] and collected data in the closed loop. This has let us project the effect of feedback onto the acquired numerical data.

The last set contains the data with Mach 0.0 and the actuator is excited with a random signal.

Such a collective training data set clearly represents how the system behaves in closed loop as well as in open loop, and how it behaves under noise driven cases and sinusoidal forcing. In all cases described above, we adopted $50 \mathrm{kHz}$ sampling rate and soft-filtered the collected data with a highpass filter having cutoff frequency equal to $10 \mathrm{kHz}$.

\section{B. Structure of the Fuzzy System}

The input to the fuzzy system at discrete time $k$ is characterized by

$$
z_{k}=\left[\begin{array}{llll}
u_{1, k} & d_{k} & u_{3, k} & M
\end{array}\right]
$$

where $M:=$ Mach number -0.3 stands for the offset removed value of the Mach number of the flow regime. The output of the fuzzy system is $x_{k+1}$ which is desired to minimize some norm of the quantity $e_{k+1}:=d_{k+1}-x_{k+1}$ over some set of observations.

The fuzzy system used in this study has therefore 4 inputs and one output. Triangular membership functions are used with product inference and singleton defuzzifier is used. The involved input variables have been quantified by linguistic labels negative $(\mathcal{N})$, zero $(\mathcal{Z})$ and positive $(\mathcal{P})$ by the membership functions defined below with a generic argument $x$. 


$$
\begin{aligned}
\mu_{\mathcal{N}}(x) & =\max \left(0, \min \left(1,-\frac{x}{L}\right)\right), \\
\mu_{\mathcal{Z}}(x) & =\max \left(0, \min \left(1-\frac{x}{L}, 1+\frac{x}{L}\right)\right), \\
\mu_{\mathcal{P}}(x) & =\max \left(0, \min \left(1, \frac{x}{L}\right)\right),
\end{aligned}
$$

where $L$ is a parameter yet to be explained. The $\mathrm{i}$-th rule of the rule base has the following form

$$
\begin{aligned}
& \mathrm{IF} u_{1, k} \text { is } \mathbb{F}_{i}^{1} \& d_{k} \text { is } \mathbb{F}_{i}^{2} \& u_{3, k} \text { is } \mathbb{F}_{i}^{3} \& M \text { is } \mathbb{F}_{i}^{4} \\
& \operatorname{THEN} f_{i} \text { is } y_{i}
\end{aligned}
$$

where $\mathbb{F}_{i}^{j}$,s denote the fuzzy sets characterized by the membership functions described in (2). The rules are enumerated and the corresponding labels for the rules $1,2, \ldots, R$ are $\mathcal{N N \mathcal { N N }}, \mathcal{N} \mathcal{N} \mathcal{N} \mathcal{Z}, \mathcal{N} \mathcal{N} \mathcal{N} \mathcal{P}, \ldots, \mathcal{P P} \mathcal{P} \mathcal{P}$, respectively. If all combinations of the rules are utilized for the three linguistic levels, we end up with $3^{4}=81$ rules in total. According to the fuzzy model described above, the firing strength of the i-th rule at time $k$ is computed as

$$
\Phi_{i, k}=\prod_{j=1}^{4} \mu_{i, \mathbb{F}_{i}^{j}}\left(z_{j, k}\right),
$$

the firing strengths at time $k$ are normalized as

$$
\phi_{i, k}=\frac{\Phi_{i, k}}{\sum_{r=1}^{R} \Phi_{r, k}},
$$

where $R$ denotes the number of rules in the rule base.

The crisp output of the used fuzzy model, i.e. $x_{k+1}$ is computed as a weighted sum of the normalized firing strengths, i.e.

$$
x_{k+1}=\sum_{r=1}^{R} y_{r} \phi_{r, k},
$$

which is desired to be an estimate for $d_{k+1}$. The choice of $y$ is explained in the next subsection.

\section{Setting up the Fuzzy Model}

At this stage, one needs to figure out the rule base and determine whether every individual rule is necessary or not. In order to do this, we have to set the membership functions first. Based on the sensory readings explained in subsection III.A, we construct a data matrix denoted by $D$, whose rows are $z_{k}$ 's (See (1)) for the experimented data acquisition conditions. The maximum and the minimum numbers obtained from the data matrix are

$$
\begin{aligned}
\min D & =\left[\begin{array}{llll}
-6.2759 & -2.0033 & -6.4205 & -0.05
\end{array}\right] \\
\max D & =\left[\begin{array}{llll}
6.6430 & 1.7253 & 6.8074 & 0.05
\end{array}\right]
\end{aligned}
$$

These values can tell the designer to decide on what range could be negative and what range could be positive or zero. According to the values in (6), the membership functions quantifying $z_{1, k}$ and $z_{3, k}$ can admit the shape parameter $L=$
4.4, those for $z_{2, k}$ and $z_{4, k}$ can be chosen as $L=0.8$ and $L=0.05$, respectively. It should be emphasized at this point that the characterization of the membership functions as well as the functions used for membership values are totally up to the designer's understanding or perception of the problem. Every different choice yields a model performing the same task to some degree of success. Due to its widespread use, we have chosen triangular membership functions.

To eliminate the unnecessary rules, define the cumulative sum square activation level of $\mathrm{r}$-th rule as follows:

$$
\zeta_{r}=\sum_{k=1}^{N} \phi_{r, k}^{2},
$$

where $N$ is the total number of samples used. Computing the above quantity for $r=1,2, \ldots, 81$, we obtain the vector of cumulative sum square activation levels as depicted in Fig. 3, where the bottom row illustrates the logarithm of the quantity seen in the top row.
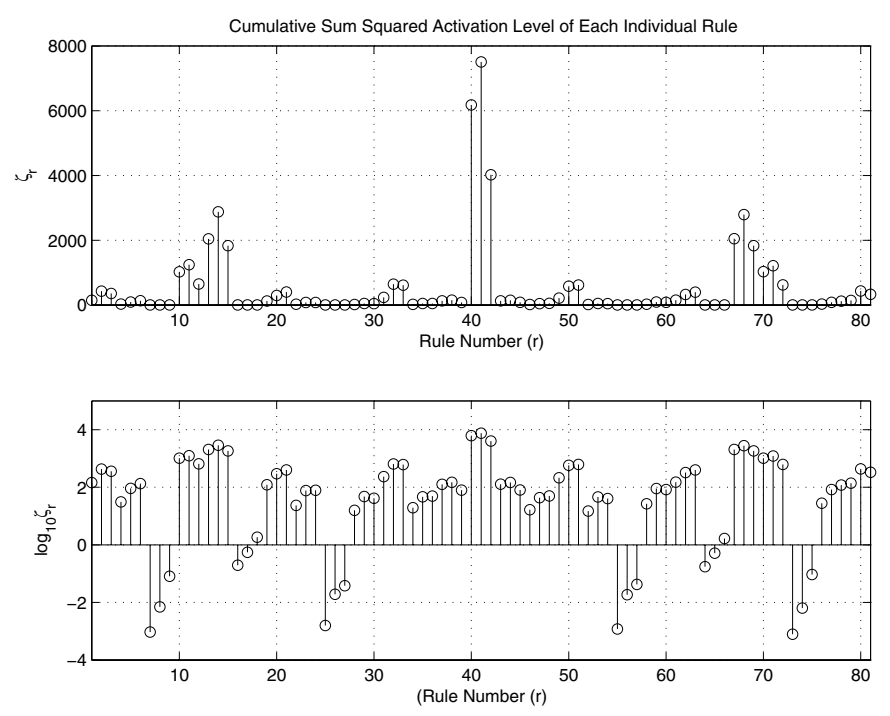

Fig. 3. A sketch of the rule activations

According to the result in Fig. 3, we infer that the rules $7,8,9,16,17,18,25,26,27,55,56,57,64,65,66$, 73,74 and 75 have the cumulative activation value less than 2 , whereas the maximum activation value is equal to 7508.3 observed for the rule $\mathcal{Z Z Z Z}$. This diagnosis lets us eliminate 18 unnecessary rules from the rule base, and the defuzzifier construction for the remaining 63 rules is performed to complete the design of the fuzzy model. In other words, $R=63$, i.e. the new cardinality of $y$.

The discussion presented so far accurately depicts the fuzzifier, which accepts the real-world variables as inputs and in response to which, a vector of normalized firing strengths is produced. Given the data in an offline manner, one can obtain the matrix of regressors by a single forward pass of the entire data set through the fuzzifier. For every pair of $\left(z_{k}, d_{k+1}\right)$, we compute one row of the regressor matrix, which is given as 


$$
\Psi=\left(\begin{array}{cccc}
\phi_{1,1} & \phi_{2,1} & \ldots & \phi_{R, 1} \\
\phi_{1,2} & \phi_{2,2} & \ldots & \phi_{R, 2} \\
\vdots & \vdots & \ddots & \vdots \\
\phi_{1, N} & \phi_{2, N} & \ldots & \phi_{R, N}
\end{array}\right)
$$

where $N$ is the total number of pairs entering the procedure. Denoting the vector of target vector, which contains the values of $d_{k+1}$ as $T$, the Least Mean Squares (LMS) estimate of the vector of defuzzifier parameters can be calculated as

$$
y=\left(\Psi^{\mathrm{T}} \Psi\right)^{-1} \Psi^{\mathrm{T}} T,
$$

which finds the value of $y$ minimizing the quadratic cost function $J=\frac{1}{2} \sum_{k=0}^{N-1}\left(d_{k+1}-x_{k+1}\right)^{2}$. In this study, we do not present any further modification on $y$ as the results of the non-adaptive case seem quite promising.

\section{Results}

In this part, we present two sets of results obtained under different regimes. In the first one, we used the data set recorded when the system was in closed loop. As illustrated in Fig. 4, the response of the fuzzy model and the obtained results show that the fuzzy model very accurately predicts $d_{k+1}$ given the vector $z_{k}$. Fast Fourier Transforms (FFT) of the two signals have been computed, and the magnitudes have been shown in the top subplot of Fig. 5, and the FFT magnitude of the difference is shown in the bottom subplot. The results indicate that the fuzzy model performs well under the specified conditions.
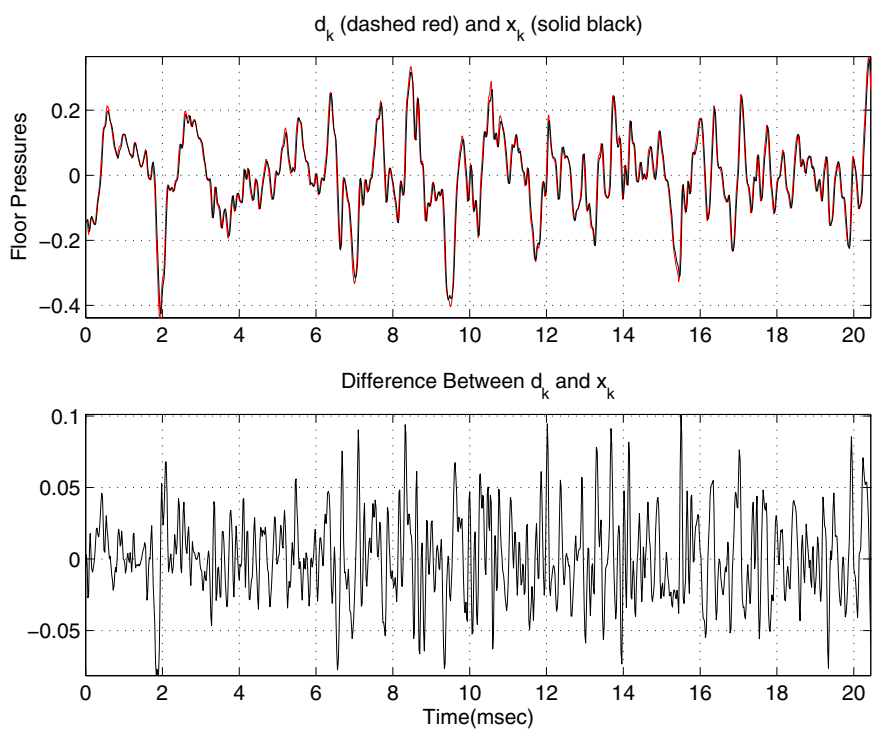

Fig. 4. The time view of the signals and the error for Mach $0.3,3920 \mathrm{~Hz}$, $4 V_{\text {rms }}$ excitation in closed loop

In the second experiment, without modifying the structure or parameters of the fuzzy model, we change the Mach number to 0.28 , excitation scheme to open-loop and excitation signal to a random sequence. The time traces are shown in Fig. 6, and the FFT plots are depicted in Fig. 7, both
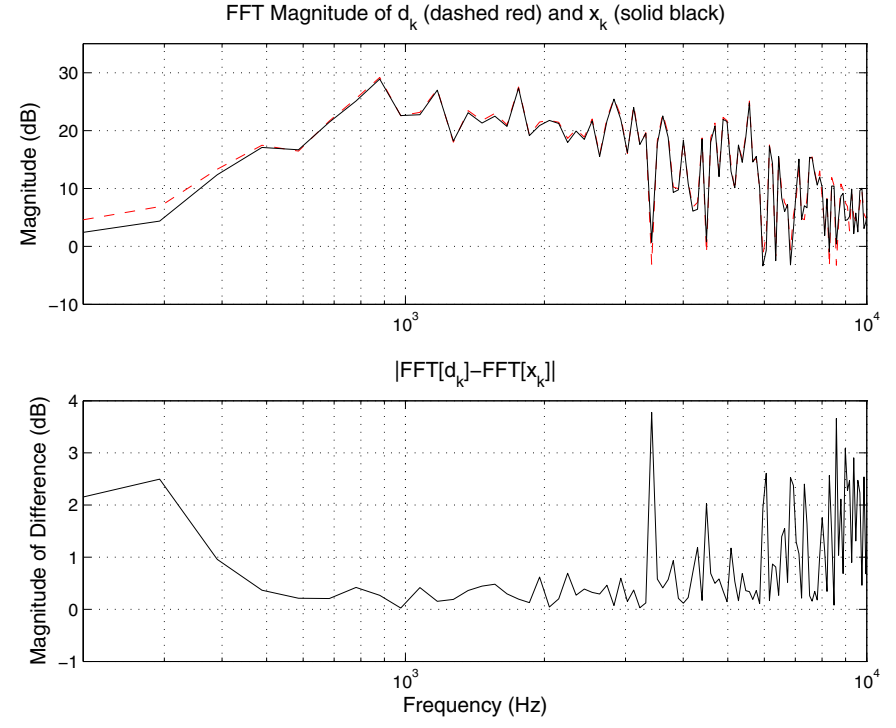

Fig. 5. Spectral view of the signals and the error for Mach 0.3, 3920Hz, $4 V_{\text {rms }}$ excitation in closed loop
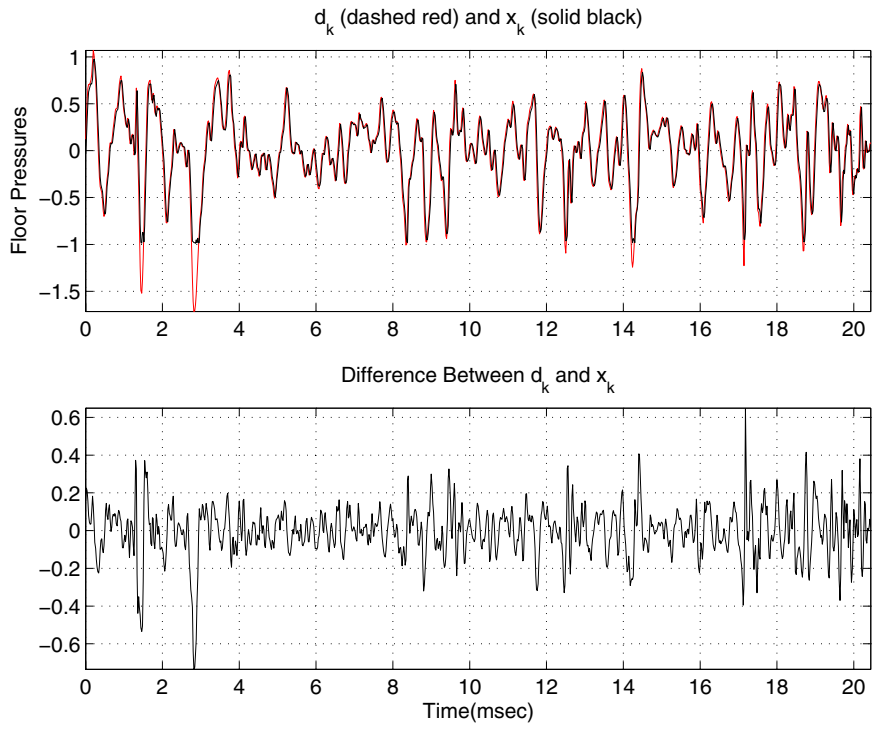

Fig. 6. The time view of the signals and the error for Mach 0.28, open loop excitation by a random sequence

emphasizing the efficiency of utilizing FL in modeling the flow past over the cavity.

In the view of these results, and those actually tested yet not presented here, the use of fuzziness in modeling of cavity flows displays some desirable properties even without adapting the model parameters.

\section{CONCLUSIONS}

This paper presents the use of FL in modeling subsonic shallow cavity lows. Under certain data acquisiton conditions, a fuzzy model has been constructed. Based on the cumulative measure of the rule activation levels, unnecessary rules have been identified and they have been eliminated from the rule base. Finally, LMS algorithm has been utilized 
FFT Magnitude of $d_{k}$ (dashed red) and $x_{k}$ (solid black)

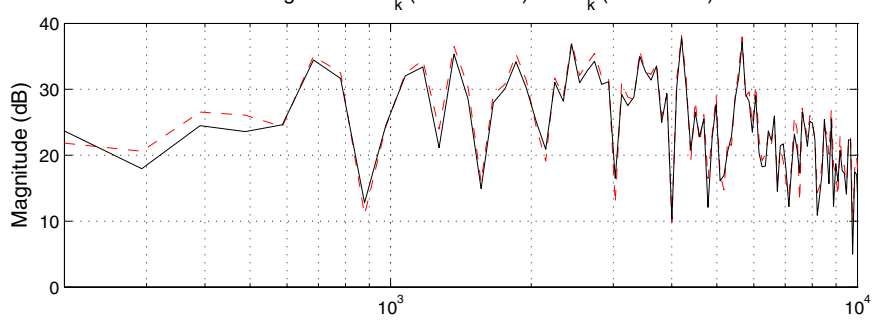

$\left|F F T\left[d_{k}\right]-F F T\left[x_{k}\right]\right|$

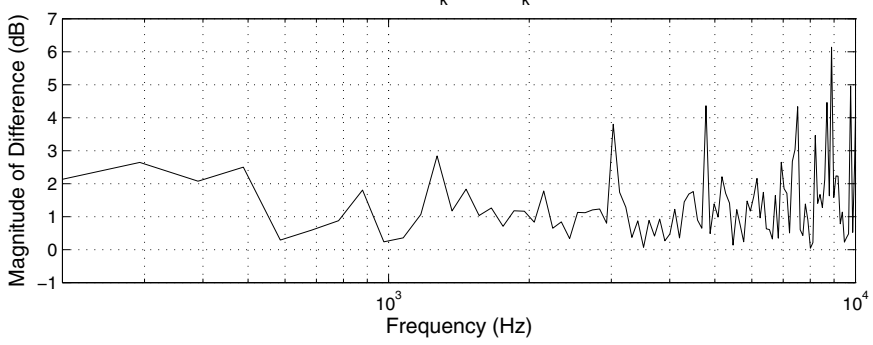

Fig. 7. Spectral view of the signals and the error for Mach 0.28, open loop excitation by a random sequence

to determine the parameters of the defuzzifier. The obtained results are quite promising in the sense of accurate prediction.

Future work on this aims to simplify the fuzzy model further and to utilize the implications in the rule base for constructing a fuzzy logic controller meeting several performance criteria.

\section{Acknowledgments}

The authors would like to thank Dr. J.H. Myatt, Dr. J. DeBonis, Dr. R.C. Camphouse, X. Yuan, E. Caraballo, J. Malone and J. Little for fruitful discussions in devising the presented work.

\section{REFERENCES}

[1] Wang, L.X., Adaptive Fuzzy Systems and Control, Design and Stability Analysis, PTR Prentice-Hall, 1994.

[2] Wang, L.X., A Course in Fuzzy Systems and Control, PTR PrenticeHall, 1997.

[3] Takagi, T., and M. Sugeno, "Fuzzy Identification of Systems and Its Applications to Modeling and Control", IEEE Transactions on Systems, Man, and Cybernetics, Vol. 15, No. 1, pp. 116-132, January 1985.

[4] Jang, J.-S. R., C.-T. Sun, E. Mizutani, Neuro-Fuzzy and Soft Computing, PTR Prentice-Hall, 1997.

[5] J. Joh, Y.-H. Chen, and R. Langari, "On the stability issues of TakagiSugeno fuzzy models," IEEE Transactions Fuzzy Systems, vol. 6, pp. 402410, Aug. 1998.

[6] Passino, K.M. and Yurkovich, S., Fuzzy Control. Reading, MA: Addison- Wesley, 1998.

[7] Cohen, K., Siegel, S. and McLaughlin, T. and Myatt, J.H., "Fuzzy Logic Control of a Circular Cylinder Vortex Shedding Model," 41th AIAA Aerospace Sciences Meeting, AIAA Paper No: 2003-1290, Reno, NV, U.S.A., 2003.

[8] Joshi, P. and Valasek, J., "Direct Comparison of Neural Network, Fuzzy Logic and Model Predictive Variable Structure Vortex Flow Controllers," Proceedings of the AIAA Guidance, Navigation and Control Conference, Portland, OR, 9-11 August 1999, (Paper No: AIAA-99-4279-CP)

[9] Dragojlovic, Z., Kaminski, D.A. and Ryoo, J., "Tuning of a Fuzzy Rule Set for Controlling Convergence of a CFD Solver in Turbulent Flow," Int. Journal of Heat and Mass Transfer, Vol.44, pp.3811-3822, 2001.
[10] Dragojlovic, Z. and Kaminski, D.A., "A Fuzzy Logic Algorithm for Acceleration of Convergence in Solving Turbulent Flow and Heat Transfer Problems," Numerical Heat Transfer, Part B, Vol.46, pp.301327, 2004.

[11] Efe, M.Ö, Yuan, X., Özbay, H., and Samimy, M., "Interpolating the Local Models of POD Using Fuzzy Decision Mechanisms," 9th Mechatronics Forum International Conference, Ankara, Turkey, Aug. 30 - Sep. 1, Ankara, Turkey, pp.347-356, 2004a.

[12] Debiasi, M., and Samimy, M., "An Experimental Study of the Cavity Flow for Closed-Loop Flow Control," AIAA Paper No: 2003-4003, June 2003.

[13] Debiasi, M. and Samimy, M., "Logic-Based Active Control of Subsonic Cavity Flow Resonance," AIAA Journal, Vol. 42, No. 9, pp. 19011909, September 2004

[14] Samimy, M., Debiasi, M., Caraballo, E., Malone, J., Little, J., Özbay, H., Efe, M.Ö., Yan, P., Yuan, X., DeBonis, J., Myatt, J.H. and Camphouse, R.C., "Exploring Strategies for Closed-loop Cavity Flow Control," 42nd AIAA Aerospace Sciences Meeting and Exhibit, January 5-8, Reno, Nevada, U.S.A., 2004, (Paper No: AIAA 2004-0576).

[15] Yan, P., Debiasi, M., Yuan, X., Caraballo, E., Efe, M.Ö., Özbay, H., Samimy, M., DeBonis, J., Camphouse, R.C., Myatt, J. H., Serrani, A., and Malone, J., "Controller Design for Active Closed-Loop Control of Cavity Flows", AIAA Paper No: 2004-0573, January 2004.

[16] Efe, M.Ö., Debiasi, M., Özbay, H., and Samimy, M., "Modeling of Subsonic Cavity Flows by Neural Networks," Int. Conf. on Mechatronics (ICM'04), June 3-5, Istanbul, Turkey, pp.560-565, 2004b.

[17] Efe, M.Ö., Debiasi, M., Yan, P., Özbay, H. and Samimy, M., "Control of Subsonic Cavity Flows by Neural Networks - Analytical Models and Experimental Validation," 43rd AIAA Aerospace Sciences Meeting and Exhibit, January 10-13, 2005, Reno, Nevada, U.S.A. (Paper No: AIAA2005-0294) 